\title{
microRNA-mRNA regulatory networks underlying chronic mucus hypersecretion in COPD
}

\author{
Hataitip Tasena ${ }^{1,2}$, Alen Faiz ${ }^{1,2,3}$, Wim Timens ${ }^{1,2}$, Jacobien Noordhoek ${ }^{1,2,3}$, \\ Machteld N. Hylkema ${ }^{1,2}$, Reinoud Gosens ${ }^{2,4}$, Pieter S. Hiemstra $\oplus^{5}$, \\ Avrum Spira ${ }^{6}$, Dirkje S. Postma ${ }^{2,3}$, Gaik W. Tew ${ }^{7}$, Michele A. Grimbaldeston ${ }^{8}$, \\ Maarten van den Berge ${ }^{2,3}$, Irene H. Heijink ${ }^{1,2,3,9}$ and Corry-Anke Brandsma $a^{1,2,9}$ \\ Affiliations: ${ }^{1}$ Dept of Pathology and Medical Biology, University of Groningen, University Medical Centre \\ Groningen, Groningen, The Netherlands. ${ }^{2}$ Groningen Research Institute for Asthma and COPD, University of \\ Groningen, University Medical Centre Groningen, Groningen, The Netherlands. ${ }^{3}$ Dept of Pulmonary Diseases, \\ University of Groningen, University Medical Centre Groningen, Groningen, The Netherlands. ${ }^{4}$ Dept of \\ Molecular Pharmacology, University of Groningen, Groningen. The Netherlands. ${ }^{5}$ Dept of Pulmonology, Leiden \\ University Medical Centre, Leiden, The Netherlands. ${ }^{6}$ Dept of Medicine, Division of Computational \\ Biomedicine, Boston University Medical Centre, Boston, MA, USA. ${ }^{7}$ Research and Early Development, \\ Genentech Inc., San Francisco, CA, USA. ${ }^{8}$ OMNI-Biomarker Development, Genentech Inc., San Francisco, CA, \\ USA. ${ }^{9}$ These authors contributed equally.
}

Correspondence: Hataitip Tasena, Dept of Pathology and Medical Biology, University Medical Centre Groningen, Hanzeplein 1 (EA10), 9700RB Groningen, The Netherlands. E-mail: h.tasenadumcg.nl

@ERSpublications

miR-134, miR-146a and the let-7 family as regulators of chronic mucus hypersecretion in COPD http://ow.ly/1RWl30kWcWF

Cite this article as: Tasena $\mathrm{H}$, Faiz A, Timens $\mathrm{W}$, et al. microRNA-mRNA regulatory networks underlying chronic mucus hypersecretion in COPD. Eur Respir J 2018; 52: 1701556 [https://doi.org/10.1183/ 13993003.01556-2017].

ABSTRACT Chronic mucus hypersecretion $(\mathrm{CMH})$ is a common feature in chronic obstructive pulmonary disease (COPD) and is associated with worse prognosis and quality of life. This study aimed to identify microRNA (miRNA)-mRNA regulatory networks underlying CMH.

The expression profiles of miRNA and mRNA in bronchial biopsies from 63 COPD patients were associated with $\mathrm{CMH}$ using linear regression. Potential mRNA targets of each CMH-associated miRNA were identified using Pearson correlations. Gene set enrichment analysis (GSEA) and STRING (search tool for the retrieval of interacting genes/proteins) analysis were used to identify key genes and pathways.

20 miRNAs and 539 mRNAs were differentially expressed with CMH in COPD. The expression of 10 miRNAs was significantly correlated with the expression of one or more mRNAs. Of these, miR-134-5p, miR-146a-5p and the let-7 family had the highest representation of CMH-associated mRNAs among their negatively correlated predicted targets. KRAS and EDN1 were identified as key regulators of CMH and were negatively correlated predicted targets of miR-134-5p and let-7a-5p, let-7d-5p, and let-7f-5p, respectively. GSEA suggested involvement of $M U C 5 A C$-related genes and several other relevant gene sets in $\mathrm{CMH}$. The lower expression of miR-134-5p was confirmed in primary airway fibroblasts from COPD patients with $\mathrm{CMH}$.

We identified miR-134-5p, miR-146a-5p and let-7 family, along with their potential target genes including KRAS and EDN1, as potential key miRNA-mRNA networks regulating CMH in COPD. 


\section{Introduction}

Chronic obstructive pulmonary disease (COPD) is a frequently occurring lung disease, associated with an abnormal inflammatory response to inhaled noxious particles and gases, including cigarette smoke. A substantial proportion of patients experience chronic cough with sputum production $[1,2]$ termed chronic bronchitis or chronic mucus hypersecretion (CMH) [3]. CMH in COPD is associated with lower quality of life, accelerated lung function decline, increased risk of exacerbations and higher mortality [1, 2, 4]. Therefore, there is an urgent need for improved treatment of $\mathrm{CMH}$ in COPD patients. Unfortunately, our current understanding of the regulatory mechanisms that drive $\mathrm{CMH}$ is still limited.

Goblet cells within the airway epithelial layer together with mucous glands in the airway submucosa are responsible for the secretion of mucins, the principal components of mucus [3]. The most abundant gel-forming mucins found in human airways are MUC5AC and MUC5B [5], which are both increased in COPD [6]. Interestingly, recent in vitro studies suggest that fibroblasts play a role in the regulation of airway epithelial mucociliary differentiation and mucus production $[7,8]$.

microRNAs (miRNAs) are small non-coding RNA molecules that target messenger RNA (mRNA), causing mRNA degradation or inhibition of protein translation [9]. Thus far, no studies have reported on differential miRNA expression in $\mathrm{CMH}$, although several miRNAs have been implicated in the response to smoking and COPD [10], including miR-146a-5p [11]. The aim of this study was to identify key miRNA-mRNA interactions underlying $\mathrm{CMH}$ in bronchial biopsies from a well-defined COPD cohort.

\section{Methods}

\section{Patient characteristics}

Baseline miRNA and mRNA expression was studied in bronchial biopsies from 63 COPD patients who participated in the Groningen and Leiden Universities Corticosteroids in Obstructive Lung Disease (GLUCOLD) study (registered at ClinicalTrials.gov with identifier number NCT00158847). Details of the study including patient characteristics were previously described $[12,13]$. Briefly, all patients had irreversible airflow limitation (post-bronchodilator forced expiratory volume in $1 \mathrm{~s}$ (FEV1) and FEV1/ inspiratory vital capacity (IVC) less than $90 \%$ CI of the predicted value) and chronic respiratory symptoms. All patients were stable, were either current or ex-smokers and were not on corticosteroid therapy. The local medical ethics committee approved the study and all patients gave their written informed consent.

\section{Definitions of chronic mucus hypersecretion}

In our dataset, clinical questionnaires were used providing information comparable to the most commonly used definition of $\mathrm{CMH}$ : symptoms of cough and phlegm on most days for more than 3 months during at least two consecutive years [4]. Thus, $\mathrm{CMH}$ was defined based on patient responses to the question "How often did you cough up sputum during the last 3 months?" (question A). Since a patient's response may vary over time, we decided to include another question "How often did you cough up sputum during the last week?" (question B), to cover both the longer time frame (3 months) and the shorter, more recent time frame (1 week). Henceforth, these were referred to as definitions A and B, respectively. A full description of the response options to these questions is presented in the supplementary material. For each question, patients were divided into three groups: no $\mathrm{CMH}$, mild $\mathrm{CMH}$ and moderate/severe $\mathrm{CMH}$.

\section{microRNAs and mRNA expression profiling}

The methods for mRNA and miRNA extraction from bronchial biopsies, for gene expression profiling using Affymetrix arrays (ThermoFisher Scientific, Santa Clara, CA, USA) and for the RNA-sequencing are described in the supplementary material.

\section{Statistical analysis}

mRNA and miRNA analyses on bronchial biopsies were performed using R software version 3.2.5 (The R Project for Statistical Computing; www.r-project.org/). A linear regression model was used to identify miRNAs and mRNAs that were differentially expressed in patients with mild or moderate/severe CMH compared to those with no $\mathrm{CMH}$. The model was corrected for age, gender, smoking history and RNA integrity number (RIN). Multiple testing correction was performed using Benjamini and Hochberg's method. Definition A was applied to acquire the primary lists of candidate miRNAs and mRNAs that were associated with $\mathrm{CMH}$ using a false discovery rate (FDR) adjusted p-value cut-off of less than 0.25. Definition $\mathrm{B}$ was then applied to further strengthen the primary findings using a nominal p-value cut-off of less than 0.05. Subsequently, the final lists consisted of candidate miRNAs and mRNAs that were associated with $\mathrm{CMH}$ according to both definitions. Other statistical tests were performed using GraphPad Prism version 6 (GraphPad Software, San Diego, USA). Differences in patient characteristics were 
compared using ANOVA. The correlation of the two CMH definitions was assessed using Spearman's rank correlation coefficient. The Mann-Whitney U-test was performed to determine significant differences in immunohistochemistry markers between $\mathrm{CMH}$ status and miRNA expression in vitro. The methods for miRNA-mRNA co-expression network analysis, gene set enrichment analysis (GSEA) and interaction network analysis using STRING, are described in the supplementary material.

microRNA expression in human primary airway epithelial cells and fibroblasts

The expression of candidate miRNAs was evaluated in air-liquid interface (ALI) differentiated primary airway epithelial cells (PAECs) and primary airway fibroblasts (PAFs) obtained during lung transplantation procedures. PAECs were obtained from six Stage IV COPD explanted lungs (supplementary table S1) and six non-COPD donor lungs as described previously [14], of which the majority did not have CMH. PAFs were isolated from Stage IV COPD patients (supplementary table S2) as described previously [15], of which eight had clinical $\mathrm{CMH}$ symptoms and eight had no clinical CMH symptoms. Cell culture procedures and reverse transcriptase quantitative PCR (RT-qPCR) details are described in the supplementary material.

\section{Results}

\section{Patient characteristics}

Microarray data of sufficient quality was obtained from 63 patients for miRNA expression profiles and from 57 patients for mRNA expression profiles. Patient characteristics are shown in table 1. CMH definitions A and B were significantly correlated ( $r=0.651, p<0.0001$; supplementary figure $S 1$ ). There was no significant difference in smoking status, smoking intensity (in pack-years), age, BMI, or lung function among the three $\mathrm{CMH}$ groups (see table 1). The study approach and flow diagram of the main findings are shown in figure 1 .

\section{CMH associated miRNAs}

To identify miRNAs associated with $\mathrm{CMH}$ we compared expression profiles of 230 miRNAs in patients with 1) mild and no $\mathrm{CMH}$ and 2) moderate/severe and no $\mathrm{CMH}$. According to definition $\mathrm{A}$, three miRNAs (miR-34b-3p, miR-92b-3p and miR-449b-5p) were higher expressed with mild $\mathrm{CMH}$, while miR-664a-5p was lower expressed. With moderate/severe $\mathrm{CMH}$, we found 39 differentially expressed miRNAs, including the four that were associated with mild $\mathrm{CMH}$ (FDR $<0.25$; figures $2 \mathrm{a}$ and $2 \mathrm{~b}$ ). According to definition B, 20 out of the 39 miRNAs were also associated with moderate/severe $\mathrm{CMH}$ and in the same direction $(\mathrm{p}<0.05)$. Among these, miR-708-5p had the highest increase (2.06) and miR-134-5p had the strongest decrease (-2.15) (supplementary table S3). No miRNA was differentially expressed in mild $\mathrm{CMH}$ according to definition $\mathrm{B}$.

\section{CMH associated mRNAs}

To identify mRNAs associated with CMH we compared expression profiles of 19793 mRNAs in patients with 1) mild and no $\mathrm{CMH}$ and 2) moderate/severe and no $\mathrm{CMH}$. According to $\mathrm{CMH}$ definition A, no differences in mRNA expression were found with mild CMH. The expression of 942 mRNAs differed with moderate/severe CMH (FDR $<0.25$; figures $2 \mathrm{c}$ and $2 \mathrm{~d}$ ). Furthermore, according to definition $\mathrm{B}, 539$ out of

\section{TABLE 1 Patient characteristics in relation to the definitions of chronic mucus hypersecretion (CMH)}

\begin{tabular}{|c|c|c|c|c|c|c|}
\hline Characteristics & \multicolumn{3}{|c|}{ CMH definition A } & \multicolumn{3}{|c|}{ CMH definition B } \\
\hline Current smokers & $4(50.0)$ & $14(63.6)$ & $23(69.7)$ & $4(50.0)$ & $17(68.0)$ & $20(67.0)$ \\
\hline Smoking intensity pack-years & $41.7(23.6-50.3)$ & $44.1(31.9-54.4)$ & $41.5(35.9-53.5)$ & $40.9(24.4-51.2)$ & $45.0(31.2-55.8)$ & $41.5(36.5-53.5)$ \\
\hline Age years & $64(57-69)$ & $59(53-64)$ & $60(57-66)$ & $56(50-60)$ & $59(55-63)$ & $62(57-69)$ \\
\hline $\mathrm{FEV}_{1} / \mathrm{FVC}$ & $0.54(0.42-0.60)$ & $0.49(0.44-0.54)$ & $0.50(0.44-0.56)$ & $0.56(0.49-0.63)$ & $0.50(0.44-0.56)$ & $0.49(0.42-0.54)$ \\
\hline RIN & $2.6(2.5-3.2)$ & $2.7(2.3-4.7)$ & $2.6(2.4-3.7)$ & $2.7(2.5-4.4)$ & $2.7(2.4-4.0)$ & $2.6(2.4-3.4)$ \\
\hline
\end{tabular}

Data are presented as $\mathrm{n}(\%)$ or median (interquartile range). BMI: body mass index; FEV1: forced expiratory volume in $1 \mathrm{~s}$; FVC: forced vital capacity; RIN: RNA integrity number. 


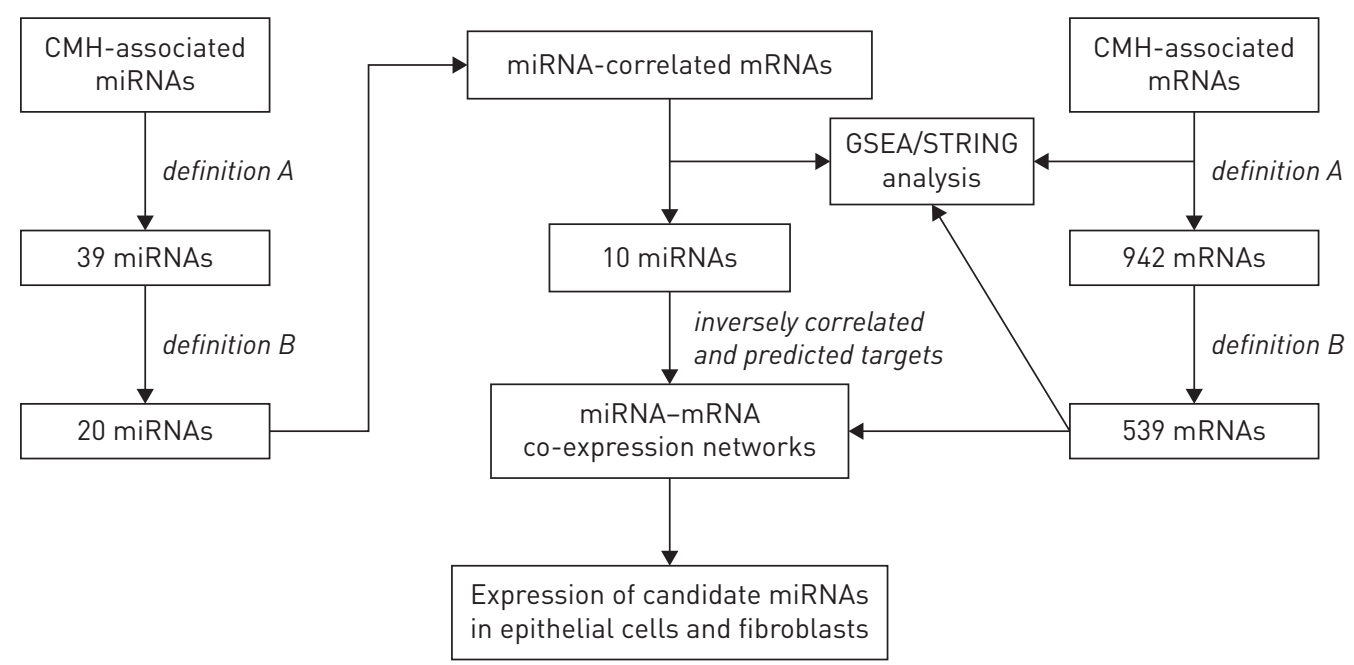

FIGURE 1 Flow diagram of the study approach. A linear regression model was used to identify chronic mucus hypersecretion (CMH) associated microRNAs (miRNAs) and $\mathrm{CMH}$-associated mRNAs based on two $\mathrm{CMH}$ definitions. Pearson correlation was used to identify miRNA-correlated mRNAs. Gene set enrichment analysis (GSEA) was performed using gene sets of interest and ranked lists of miRNA-correlated and $\mathrm{CMH}$-associated mRNAs. Interaction network analysis using STRING (search tool for the retrieval of interacting genes/ proteins) was performed on the list of $\mathrm{CMH}$-associated mRNAs correlated with at least one $\mathrm{CMH}$-associated miRNA. The list of $\mathrm{CMH}$-associated mRNAs and the lists of negatively correlated predicted targets of the candidate miRNAs were used to create miRNA-mRNA co-expression networks. Finally, the expression of candidate miRNAs was assessed in primary airway epithelial cells and primary airway fibroblasts.

942 mRNAs were also associated with $\mathrm{CMH}$ in the same direction $(\mathrm{p}<0.05)$. Among these, $264 \mathrm{mRNAs}$ were higher expressed and 275 mRNAs were lower expressed with moderate/severe CMH (supplementary table S4). The top 20 significant mRNAs are shown in table 2.

To validate the findings from the microarray dataset, RNA-sequencing was performed on bronchial biopsies from a subset of the patients in the GLUCOLD cohort with moderate/severe $\mathrm{CMH}(\mathrm{n}=21)$ and without $\mathrm{CMH}(\mathrm{n}=5)$. GSEA was performed to assess if the $\mathrm{CMH}$-associated genes in our microarray dataset were enriched among the $\mathrm{CMH}$-associated genes in our RNA-sequencing dataset. Indeed, we found a significant enrichment (supplementary figure S2 and supplementary table S7).

To ensure that the observed differences in miRNA and mRNA expression were not due to changes in cellular composition, we analysed available immunohistochemical data on the numbers of neutrophils, macrophages, eosinophils, CD3+/CD4+/CD8+ lymphocytes, mast cells and epithelial cells in the bronchial biopsies previously described [13]. We observed that only CD8+ lymphocytes differed with $\mathrm{CMH}$. When accounting for the CD8+ cell number in our linear regression model, all $20 \mathrm{CMH}$-associated miRNAs and $539 \mathrm{CMH}$-associated mRNAs remained significant.

Gene set enrichment analysis of CMH-associated mRNAs

GSEA was performed to identify pathways, biological processes and molecular functions in which the $\mathrm{CMH}$-associated mRNAs may be involved. The top 20 significant gene sets enriched among mRNAs differentially expressed with $\mathrm{CMH}$ according to both definitions are shown in table 3. Among these were gene sets related to cilium development and function, neurohormonal signalling, ion channel activities and extracellular matrix $(\mathrm{ECM})$ structure. The complete list of significant gene sets (FDR $<0.01)$ is reported in supplementary table S5. Furthermore, we investigated whether our $\mathrm{CMH}$-associated mRNAs are involved in the mechanisms relevant to MUC5AC expression. Since MUC5AC was not expressed above background levels in our microarrays, we built further on the findings of WANG et al. [16] who previously identified 73 MUC5AC-associated core genes which were higher expressed in small airway epithelium from individuals with high MUC5AC gene expression versus those with low MUC5AC gene expression. Using this list, we found a strong enrichment of $M U C 5 A C$-associated genes among the genes higher expressed with $\mathrm{CMH}$ according to both definition A (enrichment score $(E S)=0.40, p<0.001)$ and definition $B(E S=0.44, p<0.001)$ (supplementary figure S3).

\section{Identification of miRNA-mRNA co-expression networks contributing to CMH in COPD}

To identify mRNAs that are regulated by the CMH-associated miRNAs, we assessed positive and negative correlations between the expression of each miRNA and the mRNA expression profile in the matched 
a)

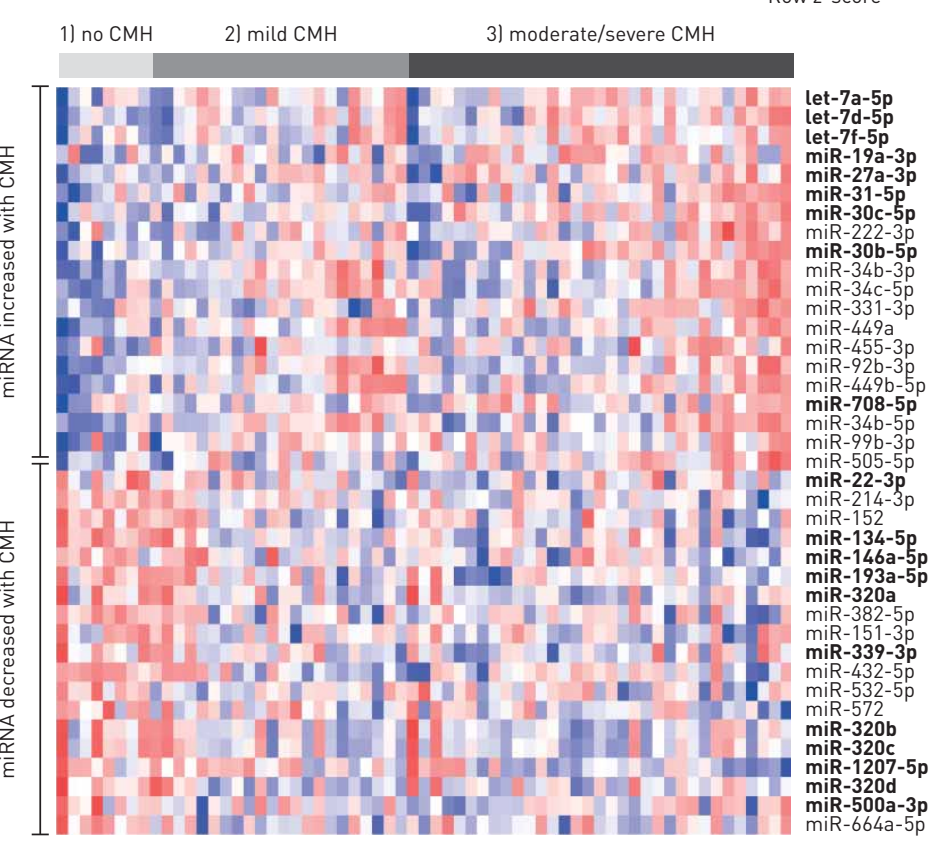

b)

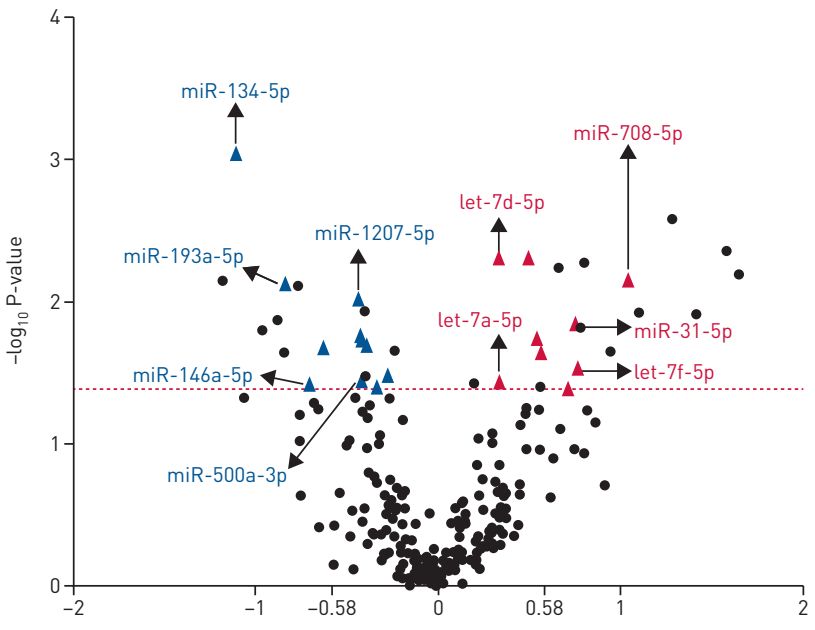

$\log _{2}$ fold change
Lower expressed with $\mathrm{CMH}$

Higher expressed with $\mathrm{CMH}$ c)

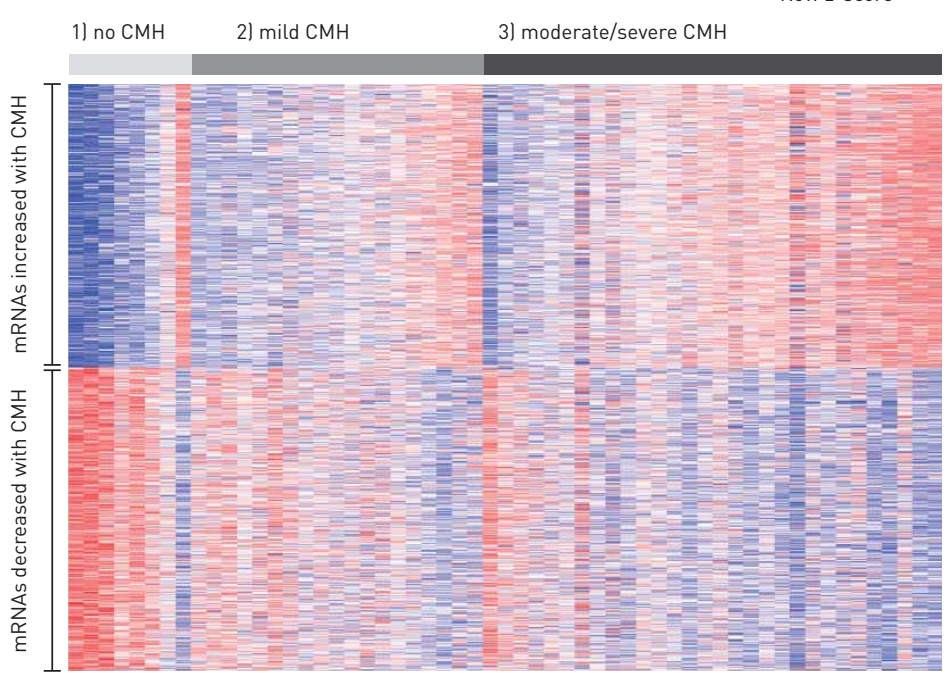

Decreased expression

Increased expressio

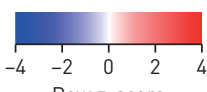

$\begin{array}{lll}-2 & 0 & 2\end{array}$

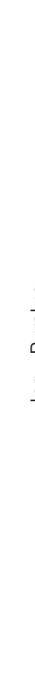

d)

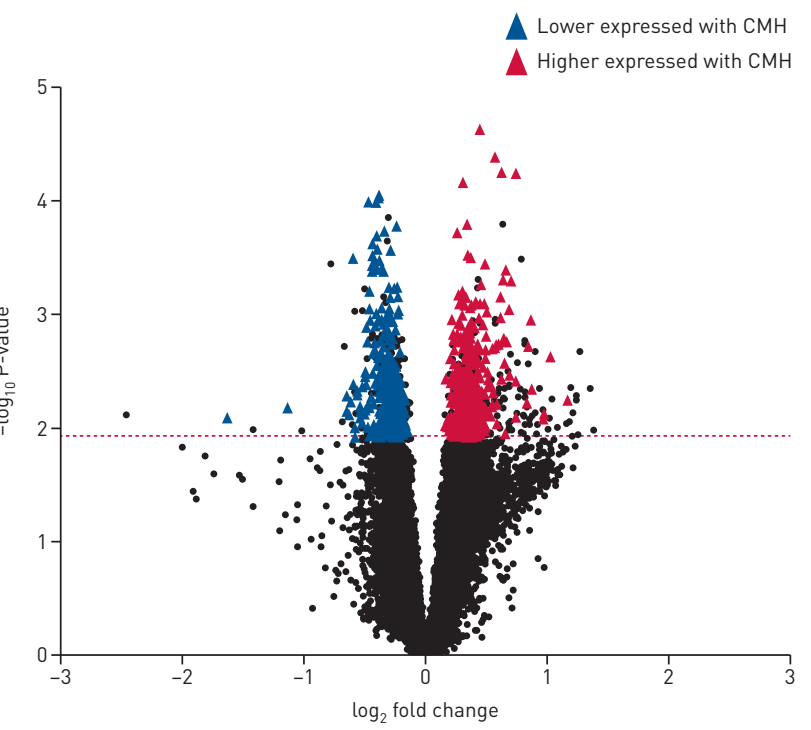

FIGURE 2 MicroRNAs (miRNAs) and mRNAs differentially expressed with chronic mucus hypersecretion (CMH). Patients were classified into three groups depending on their $\mathrm{CMH}$ status, as defined by two definitions, with miRNA profiles of patients with moderate/severe $\mathrm{CMH}$ being compared to those of patients with no $\mathrm{CMH}$. Heat map (a) shows expression changes of $39 \mathrm{miRNAs}$ in the patients with moderate/severe $\mathrm{CMH}$ ( $\mathrm{n}=33$ ) compared to those with no $\mathrm{CMH}(\mathrm{n}=8)$ according to definition A. Bold labels indicate the 20 miRNAs whose expression was significant based on both definition $\mathrm{A}$ and definition B. Volcano plot (b) shows expression changes of 230 miRNAs in the patients with moderate/severe $\mathrm{CMH}$ compared to those with no $\mathrm{CMH}$ according to definition A. Heat map (c) shows expression changes of $942 \mathrm{mRNAs}$ in the patients with severe $\mathrm{CMH}$ ( $\mathrm{n}=30$ ) compared to those with no $\mathrm{CMH}(\mathrm{n}=8)$ according to definition A. Volcano plot (d) shows expression changes of 19793 mRNAs in the patients with severe $\mathrm{CMH}$ compared to those with no $\mathrm{CMH}$ according to definition A. Blue indicates significant miRNAs/mRNAs lower expressed with $\mathrm{CMH}$ while red represents significant miRNAs/mRNAs higher expressed with $\mathrm{CMH}$. Triangles represent miRNAs/mRNAs of which differential expression was significant based on both definitions. A false-discovery rate (FDR) $<0.25$ was a cut-off for definition $A$ and a nominal $p$-value $<0.05$ was a cut-off for definition B.

biopsies (same patient). The expression levels of 10 out of 20 miRNAs (i.e. let-7a-5p, let-7d-5p, let-7f-5p, miR-31-5p, miR-708-5p, miR-134-5p, miR-146a-5p, miR-193-5p, miR-500a-3p and miR-1207-5p) were significantly correlated with at least one mRNA (FDR <0.25; supplementary table S6). 
TABLE 2 The top 20 significant mRNAs differently expressed with chronic mucus hypersecretion $(\mathrm{CMH})$ and their statistics

\begin{tabular}{|c|c|c|c|c|c|c|c|}
\hline \multirow[t]{2}{*}{ Gene } & \multicolumn{4}{|c|}{$\begin{array}{c}\text { Moderate/severe } \mathrm{CMH} \text { versus no } \mathrm{CMH} \\
\text { (definition A) }\end{array}$} & \multicolumn{3}{|c|}{$\begin{array}{c}\text { Moderate/severe CMH versus no } \\
\text { CMH (definition B) }\end{array}$} \\
\hline & t-value & FC & p-value & FDR & t-value & FC & p-value \\
\hline AIG1 & 4.667 & 1.355 & $2.32 \mathrm{E}-05$ & 0.225 & 2.548 & 1.213 & $1.40 \mathrm{E}-02$ \\
\hline LRRC8B & 4.498 & 1.487 & 4.10E-05 & 0.225 & 3.117 & 1.359 & $3.03 E-03$ \\
\hline ROD1 & 4.410 & 1.538 & $5.49 \mathrm{E}-05$ & 0.225 & 3.515 & 1.462 & $9.43 E-04$ \\
\hline OSBPL3 & 4.402 & 1.671 & $5.64 \mathrm{E}-05$ & 0.225 & 3.130 & 1.522 & $2.91 \mathrm{E}-03$ \\
\hline $\mathrm{RC} 3 \mathrm{H} 1$ & 4.345 & 1.238 & $6.82 \mathrm{E}-05$ & 0.225 & 2.760 & 1.173 & 8.07E-03 \\
\hline$R Q C D 1$ & 4.082 & 1.264 & $1.61 \mathrm{E}-04$ & 0.231 & 4.512 & 1.316 & $3.91 \mathrm{E}-05$ \\
\hline LIAS & 4.030 & 1.194 & $1.90 \mathrm{E}-04$ & 0.231 & 2.254 & 1.124 & $2.86 \mathrm{E}-02$ \\
\hline USP46 & 3.884 & 1.271 & $3.02 \mathrm{E}-04$ & 0.231 & 2.608 & 1.194 & $1.20 \mathrm{E}-02$ \\
\hline MTF1 & 3.873 & 1.289 & $3.13 E-04$ & 0.231 & 2.842 & 1.227 & $6.47 E-03$ \\
\hline LOC100133388 & -4.265 & -1.306 & $8.86 \mathrm{E}-05$ & 0.225 & -2.072 & -1.173 & 4.34E-02 \\
\hline NKD1 & -4.249 & -1.312 & $9.36 \mathrm{E}-05$ & 0.225 & -2.552 & -1.198 & $1.38 \mathrm{E}-02$ \\
\hline FAM115A & -4.223 & -1.386 & $1.02 \mathrm{E}-04$ & 0.225 & -2.518 & -1.266 & $1.50 \mathrm{E}-02$ \\
\hline C1QTNF1 & -4.222 & -1.328 & $1.02 \mathrm{E}-04$ & 0.225 & -3.233 & -1.270 & $2.17 \mathrm{E}-03$ \\
\hline RPL23AP64 & -4.075 & -1.183 & $1.65 \mathrm{E}-04$ & 0.231 & -2.915 & -1.142 & 5.30E-03 \\
\hline WTIP & -4.039 & -1.266 & $1.84 \mathrm{E}-04$ & 0.231 & -2.435 & -1.167 & $1.85 \mathrm{E}-02$ \\
\hline LIMS2 & -4.010 & -1.326 & $2.02 \mathrm{E}-04$ & 0.231 & -4.282 & -1.342 & $8.38 \mathrm{E}-05$ \\
\hline BTN2A3 & -3.961 & -1.355 & $2.37 \mathrm{E}-04$ & 0.231 & -2.889 & -1.296 & $5.70 \mathrm{E}-03$ \\
\hline PDGFB & -3.929 & -1.321 & $2.62 \mathrm{E}-04$ & 0.231 & -3.030 & -1.262 & $3.87 \mathrm{E}-03$ \\
\hline C15orf60 & -3.916 & -1.223 & $2.73 E-04$ & 0.231 & -2.902 & -1.180 & $5.50 \mathrm{E}-03$ \\
\hline EDN1 & -3.884 & -1.357 & $3.02 \mathrm{E}-04$ & 0.231 & -2.098 & -1.207 & 4.10E-02 \\
\hline
\end{tabular}

t-value: a statistic reflecting the size difference relative to its standard error; FC: fold change; FDR: false discovery rate.

To identify potential direct targets, the lists of mRNAs negatively correlated with miRNAs were compared to the list of the miRNAs' predicted targets. The mRNAs that overlapped in both lists were used to generate miRNA-mRNA co-expression networks (figure 3a). Thus, let-7a-5p, let-7d-5p, let-7f-5p, miR-31-5p and miR-708-5p, which were higher expressed with $\mathrm{CMH}$, shared several potential targets and, as expected, the members of let-7 family clustered together through their shared target genes. Similarly, miR-134-5p, miR-146a-5p, miR-500a-3p and miR-1207-5p, which were lower expressed with CMH, shared several potential targets (figure 3a). In these networks, the interactions between miRNAs and negatively correlated predicted targets that were also associated with $\mathrm{CMH}$ (indicated in figure $3 \mathrm{a}$ ) are of special interest as these are the potential key drivers of $\mathrm{CMH}$. Among the higher expressed miRNAs, the let-7 cluster represents a key cluster with negative correlation with $16 \mathrm{CMH}$-associated potential targets. Among the lower expressed miRNAs, miR-134-5p and miR-146a-5p are key miRNAs negatively correlating with eight and 10 $\mathrm{CMH}$-associated potential targets, respectively. The percentage of $\mathrm{CMH}$-associated potential targets among the negatively correlated predicted targets was highest for miR-134-5p (figure 3a).

To identify potential interactions among $\mathrm{CMH}$-associated mRNAs, interaction network analysis was performed using STRING (figure 3b). The networks point towards KRAS, EDN1, PRKAR2A, GSK3B and $P O L R 2 H$ as hub genes and potential regulators of $\mathrm{CMH}$ in our biopsies, as they possessed the most interactions with other genes. Interestingly, KRAS and EDN1 are potential targets of miR-134-5p and let-7a-5p/let-7d-5p/let-7f-5p, respectively. There was also clear clustering of several collagen and other ECM-related genes.

\section{Enrichment of CMH- and MUC5AC-associated genes among the miRNA-correlated genes}

GSEA revealed that genes higher expressed with $\mathrm{CMH}$ were enriched among the genes positively correlated with miRNAs higher expressed with $\mathrm{CMH}$ and among the genes negatively correlated with miRNAs lower expressed with $\mathrm{CMH}$, and vice versa for the genes that were lower expressed with $\mathrm{CMH}$ (figures $4 \mathrm{a}, 4 \mathrm{~b}$ and supplementary figure $\mathrm{S} 4$ ).

We found that the MUC5AC-associated gene set [16] was significantly enriched among the genes positively correlated with miRNAs that were higher expressed with $\mathrm{CMH}$ and among the genes negatively correlated with miRNAs that were lower expressed with CMH, except for miR-193a-5p (figure 4c and supplementary figure S5). 
TABLE 3 The top 20 significant gene sets enriched among chronic mucus hypersecretion (CMH) associated mRNAs

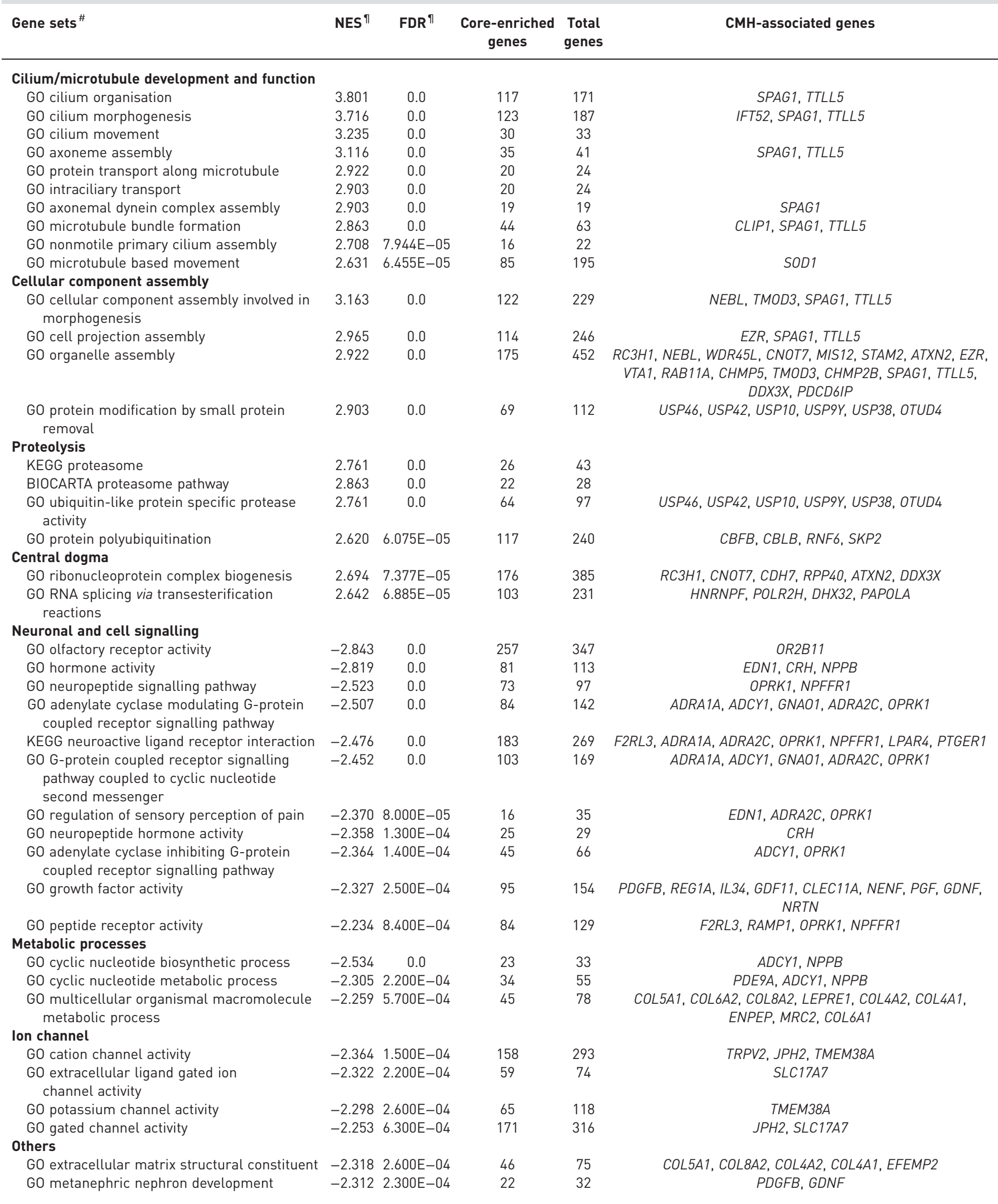

NES: normalised enrichment score; FDR: false discovery rate; GO: gene ontology; KEGG: Kyoto Encyclopedia of Genes and Genomes. ${ }^{\#}$ : gene sets enriched among genes higher expressed with $\mathrm{CMH}^{\text {१ }}{ }^{\text {: }}$ statistics based on mRNAs associated with CMH definition A. 
a)

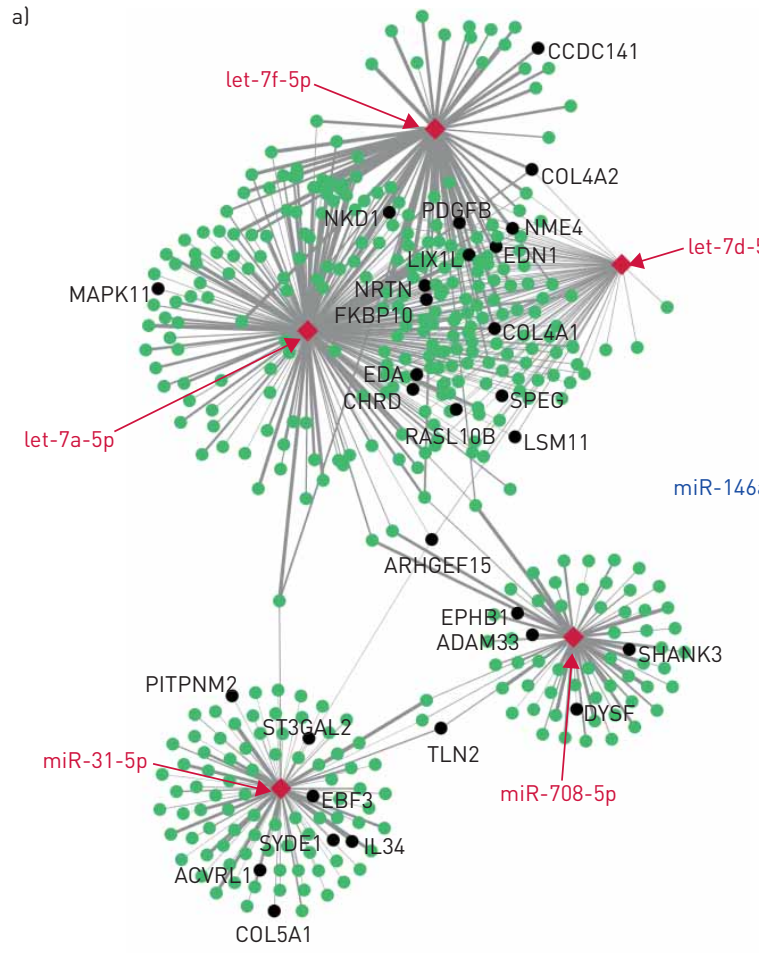

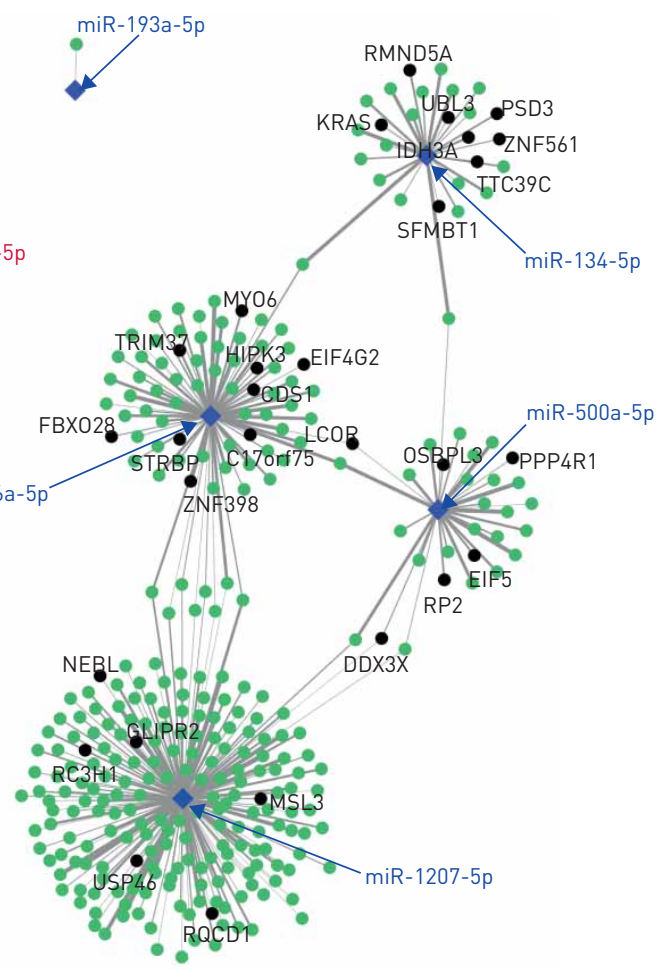

\begin{tabular}{cccccccccc}
\hline & let-7a-5p & let-7d-5p & let-7f-5p & miR-31-5p & miR-708-5p & miR-134-5p & miR-146a-5p & miR-193a-5p miR-500a-3p & miR-1207-5p \\
\cline { 2 - 8 } & 6.5 & 11.4 & 10.3 & 9.6 & 7.7 & 29.6 & 13.0 & 0.0 & 20.0 \\
\hline
\end{tabular}

\#Representation of $\mathrm{CMH}$-associated genes in all potential targets of each miRNA

b)

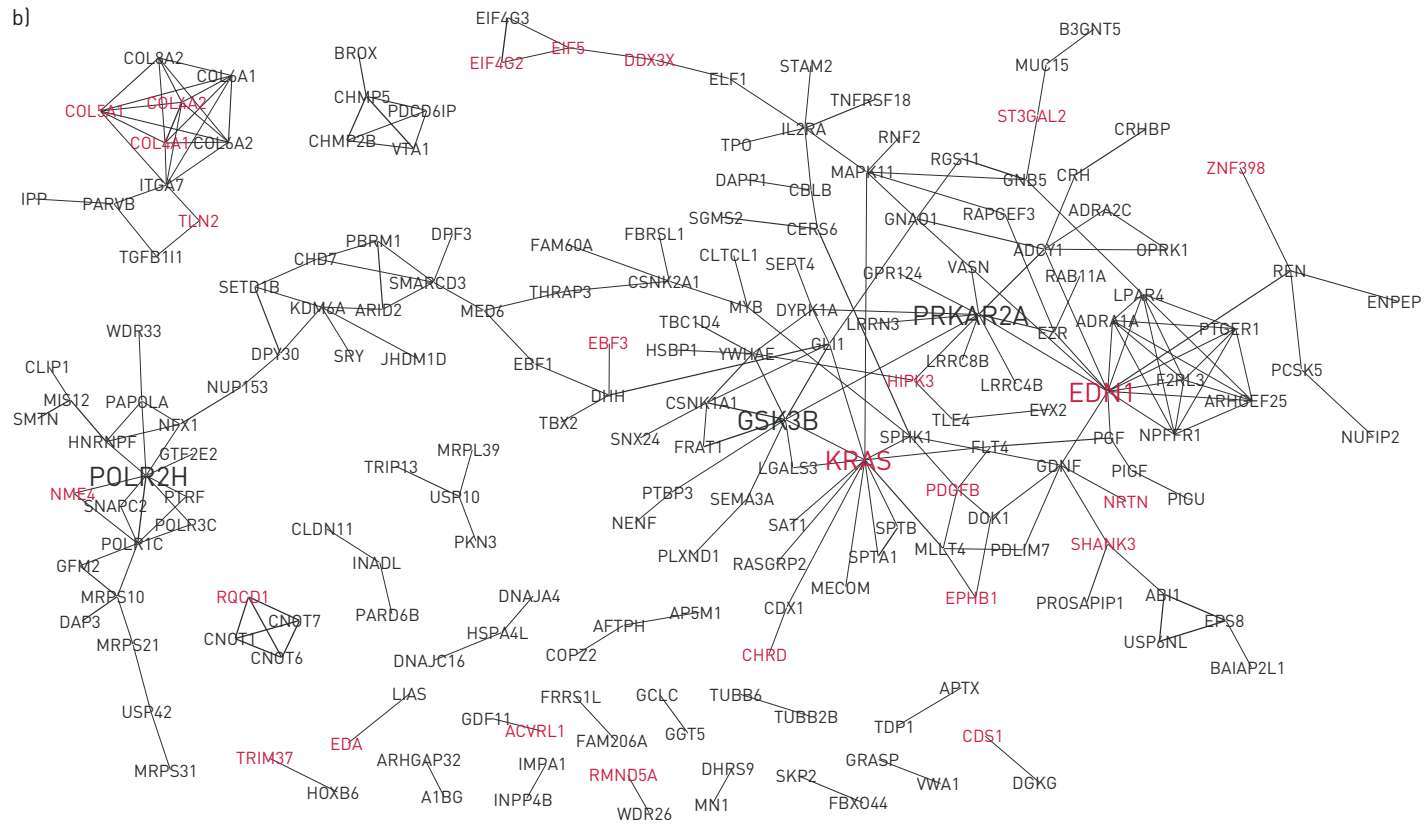

FIGURE 3 Potential networks underlying chronic mucus hypersecretion (CMH) in chronic obstructive pulmonary disease (COPD). MicroRNA (miRNA)-mRNA co-expression networks are shown in part (a). Red diamonds represent miRNAs higher expressed with CMH while blue diamonds represent miRNAs lower expressed with $\mathrm{CMH}$. Green circles represent predicted target genes negatively correlated with a particular miRNA while black circles represent negatively correlated predicted targets of the miRNAs whose expression was also associated with $\mathrm{CMH}$. Line width correlates to degree of significance of the miRNA-mRNA correlation. The percentage of CMH-associated genes among the negatively correlated predicted targets of each miRNA is illustrated in the table. Predicted interactions among CMH-associated targets of CMH-associated miRNAs are shown in part (b). The networks were created using the STRING (search tool for the retrieval of interacting genes/proteins) database. All mRNAs

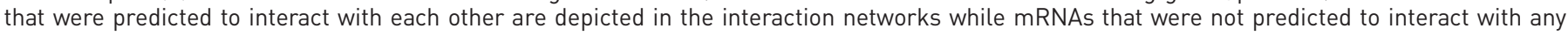
other mRNA were excluded. Red text represents negatively correlated predicted targets of miRNAs. Large and bold text represents the top five

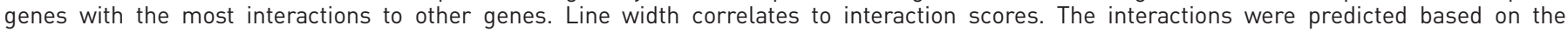
combined score of all active interaction sources with the minimum required interaction score of 0.700 . Only connected nodes are shown. 
miR-1207-5p

miR-500a-3p

miR-193a-5p

miR-146a-5p

miR-134-5p

miR-708-5p

$\operatorname{miR}-31-5 p$

let-7f-5p

let-7d-5p

let-7a-5p

b)

miR-1207-5p

miR-500a-3p

miR-193a-5p

miR-146a-5p

miR-134-5p

miR-708-5p

miR-31-5p

let-7f-5p

let-7d-5p

let-7a-5p

c)

miR-1207-5p

miR-500a-3p

miR-193a-5p

miR-146a-5p

miR-134-5p

miR-708-5p

miR-31-5p

let-7f-5p

let- $7 d-5 p$

let-7a-5p

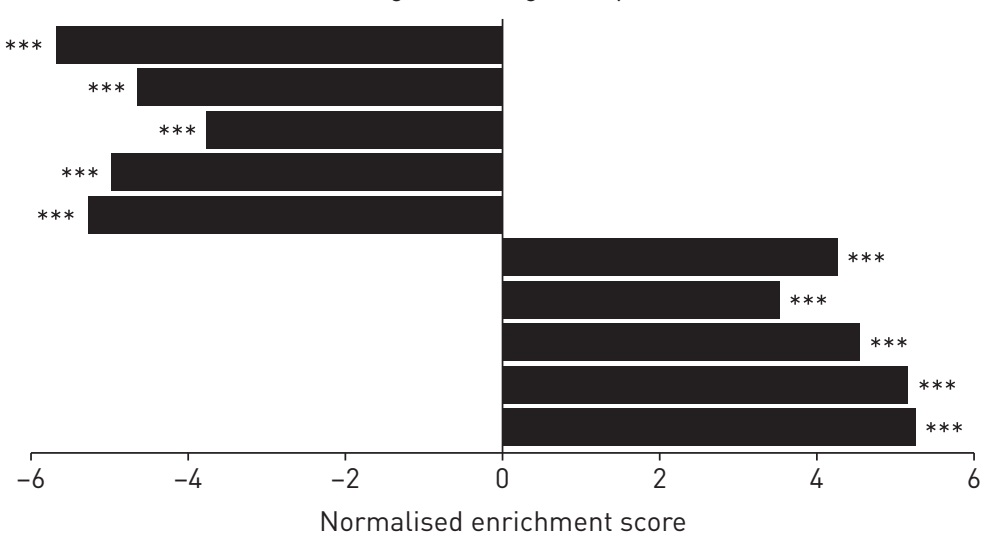

Enrichment of the gene set lower expressed with $\mathrm{CMH}$
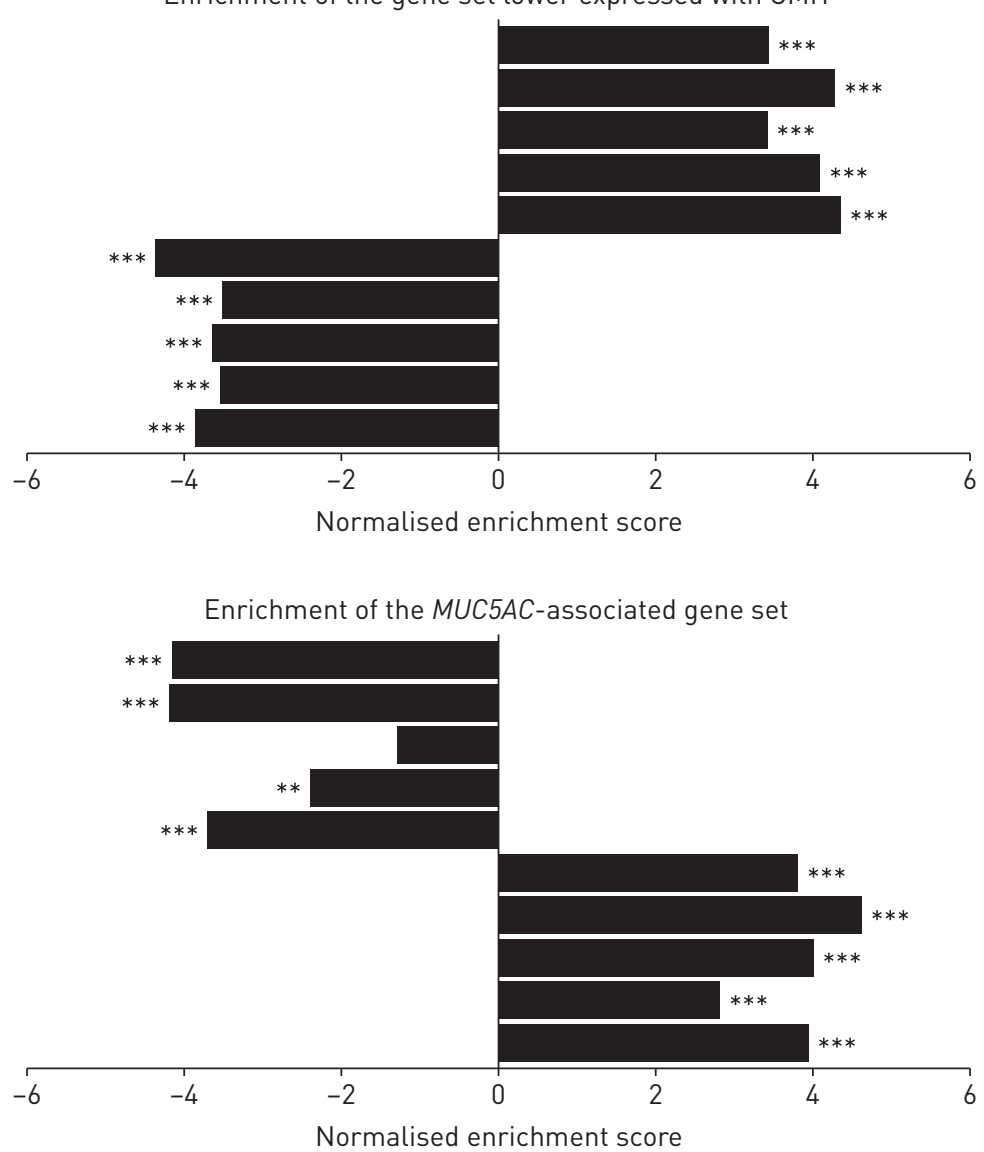
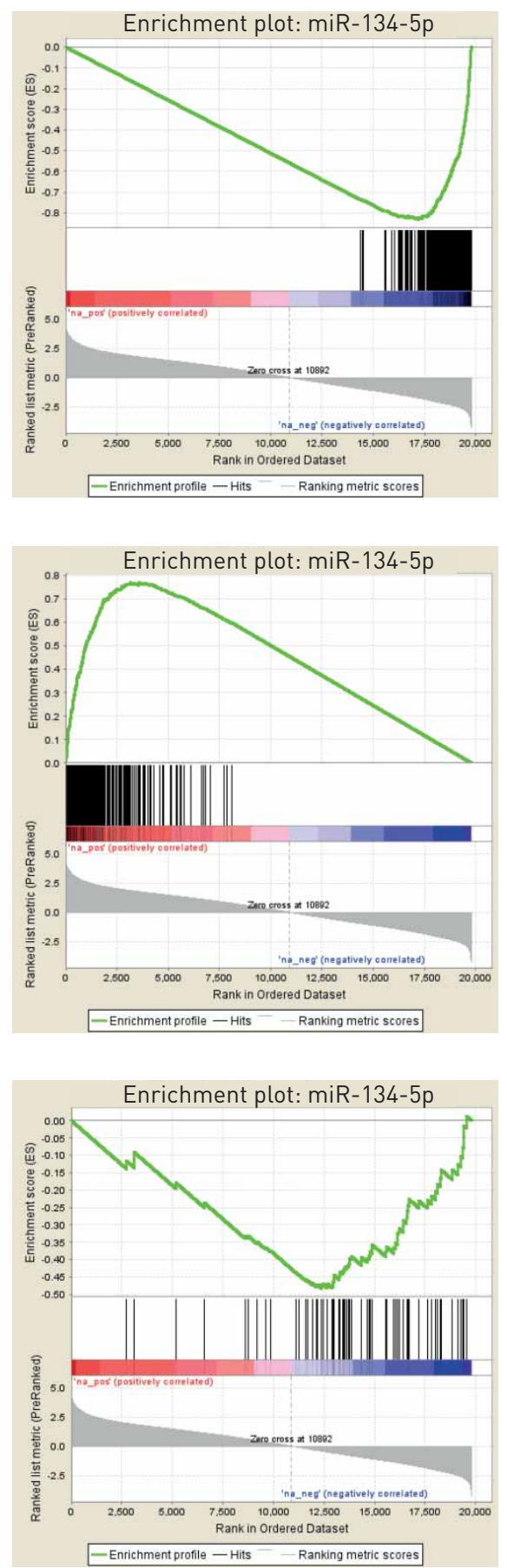

FIGURE 4 Overrepresentation of chronic mucus hypersecretion (CMH) associated genes and MUC5AC-associated genes among microRNA (miRNA) correlated genes. Enrichment of genes higher expressed with $\mathrm{CMH}$ among the genes correlated with $\mathrm{CMH}$-associated miRNAs is shown in part (a). Enrichment of genes lower expressed with $\mathrm{CMH}$ among the genes correlated with $\mathrm{CMH}$-associated miRNAs is shown in part (b). Enrichment of MUC5AC-associated genes among the genes correlated with $\mathrm{CMH}$-associated miRNAs is shown in part (c). The enrichment plots of miR-134-5p are shown as an example. Asterisks represent significant enrichment. ${ }^{* *}: p<0.01 ;{ }^{* * *}: p<0.001$.

\section{Expression of candidate miRNAs and mRNAs in human primary airway epithelial cells and fibroblasts}

As bronchial biopsy specimens contain various cell types, including mucus producing epithelial cells, we investigated whether the CMH-associated miRNAs identified in biopsies were expressed in PAECs in vitro. ALI-differentiated PAECs expressed let-7a-5p (representative of the let-7 family), miR-31-5p, miR-708-5p, miR-146a-5p and miR-193-5p (figure 5a), but not miR-134-5p, miR-500a-3p and miR-1207-5p 
a)

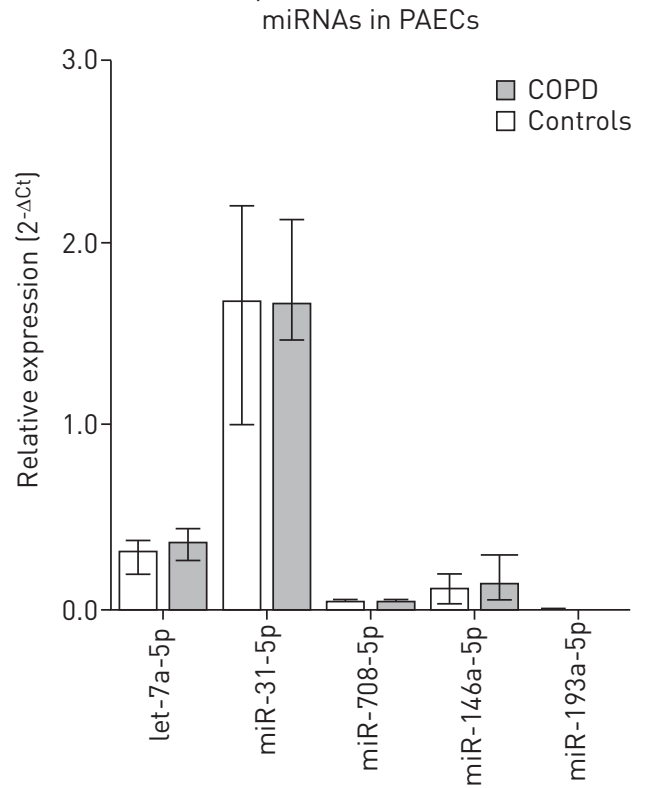

b)

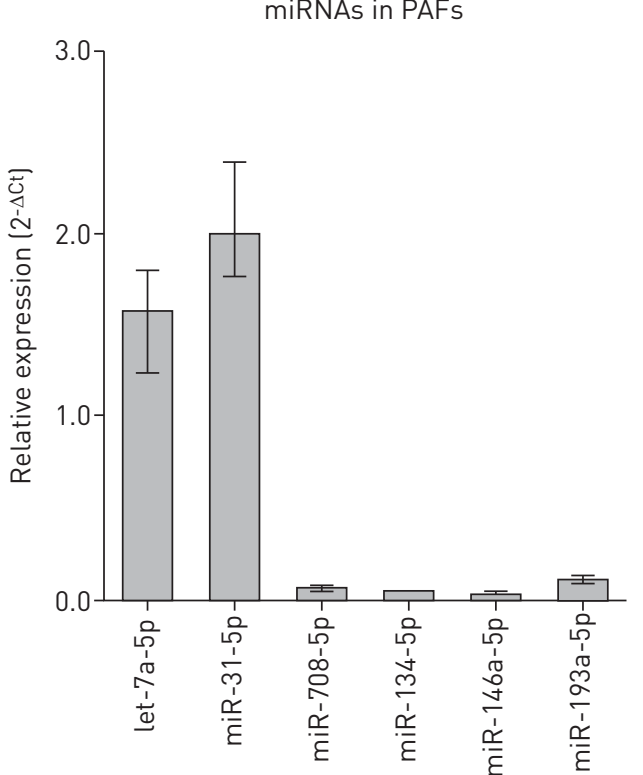

c)

miR-134-5p expression in PAFs

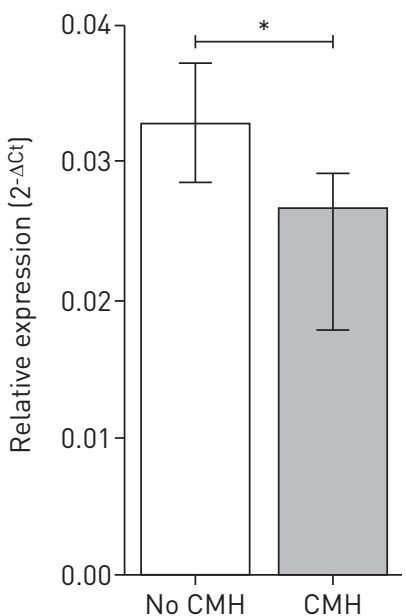

FIGURE 5 Expression of candidate microRNAs (miRNAs) in primary human airway structural cells. miRNAs expressed in primary airway epithelial cells (PAECs) are shown in part (a). PAECs were derived from chronic obstructive pulmonary disease (COPD) patients ( $\mathrm{n}=6$ ) and healthy controls $(n=6)$ and grown at an air-liquid interface for 14 days. Cells were hormonally deprived overnight and RNA was isolated. miRNAs expressed in primary airway fibroblasts (PAFs) from COPD patients $(n=16)$ are shown in part (b). The differential expression of miR-134-5p in PAFs from COPD patients with chronic mucus hypersecretion (CMH) compared to those with no $\mathrm{CMH}$ is shown in part (c). PAFs were derived from COPD patients with $\mathrm{CMH}(\mathrm{n}=8)$ and without $\mathrm{CMH}(\mathrm{n}=8)$ and grown until confluent. Cells were then serum deprived for $24 \mathrm{~h}$ and RNA was isolated. Expression of all miRNAs was normalised to RNU48. Relative expression levels $\left(2^{-\Delta \mathrm{Ct}}\right.$, where $\Delta \mathrm{Ct}$ equals the cycle threshold (Ct) of the miRNA of interest minus the Ct of RNU48) are shown as median \pm interquartile range. *: $\mathrm{p}<0.05$ between the indicated values (as assessed by the Mann-Whitney U-test).

(cycle threshold $(\mathrm{Ct})>35$ ). We did not find any differences in miRNA expression between COPD and healthy control-derived PAECs (figure 5a), neither did we observe a significant difference in MUC5AC expression between these two groups (supplementary figure S6).

As our previous report suggests involvement of fibroblasts in mucus production via crosstalk with epithelial cells [8], we additionally investigated candidate miRNA expression in PAFs derived from COPD patients with or without clinical CMH symptoms. All PAFs expressed let-7a-5p, miR-31-5p, miR-708-5p, miR-134-5p, miR-146a-5p and miR-193a-5p (figure 5b), but not miR-500a-3p and miR-1207-5p. Of interest, the expression of miR-134-5p (figure $5 \mathrm{c}$ ), but not the other miRNAs, was significantly lower in PAFs derived from the patients with $\mathrm{CMH}$, in line with the findings in our biopsies.

We used publicly available gene expression profiles [17] to assess whether the top $20 \mathrm{CMH}$-associated genes were expressed in ALI-differentiated PAECs and found that the majority of them were (supplementary figure S7). It should be noted, however, that LOC100133388, RPL23AP64 and C15orf60 were not available in this dataset. Furthermore, using publicly available gene expression profiles of primary human lung fibroblasts [18], we found that the majority of these genes were also expressed in fibroblasts, with the exception of NKD1 and PDGFB (supplementary figure S8). Again, LOC100133388 was not available in this dataset.

\section{Discussion}

We identified 20 miRNAs and 539 mRNAs associated with CMH in bronchial biopsies from COPD patients. Our data suggest that miR-134-5p, miR-146a-5p and the let-7 family may regulate CMH via their potential $\mathrm{CMH}$-associated targets (e.g. KRAS and EDN1). The relevance of our CMH signatures was supported by the enrichment of MUC5AC-associated genes [16] among our CMH-associated mRNAs and mRNAs that correlated with the $\mathrm{CMH}$-associated miRNAs. Furthermore, our in vitro studies demonstrated that miR-134-5p was lower expressed in PAFs from COPD patients with $\mathrm{CMH}$ compared to those without $\mathrm{CMH}$, which is in line with the findings in bronchial biopsies and supports a key role for miR-134-5p in regulating $\mathrm{CMH}$ in $\mathrm{COPD}$.

Since we had both miRNA and mRNA data available from the same bronchial biopsies, we were able to create miRNA-mRNA co-expression networks. This analysis identified the let-7 family, miR-134-5p and 
miR-146a-5p with their CMH-associated targets to be the key regulators of CMH. Notably, miR-134-5p had the highest percentage of $\mathrm{CMH}$-associated genes among its potential targets, of which KRAS was identified as a hub and potential regulatory gene for $C M H$. KRAS was previously found to be one of the MUC5AC-associated core genes [16]. Interestingly, the expression of miR-134-5p was lower in fibroblasts from COPD patients with $\mathrm{CMH}$ than those without, in line with the finding in biopsies. It was not expressed in epithelial cells, suggesting an indirect regulation of $\mathrm{CMH}$ via fibroblasts. There are various explanations why the differential expression of miR-134-5p in fibroblasts can affect MUC5AC-associated genes identified in airway epithelium. First, miRNAs can be secreted into exosomes [19] and thus transported from one cell type (e.g. fibroblasts) to another (e.g. epithelium). Secondly, MUC5AC-associated genes are not only expressed by epithelial cells, but also by fibroblasts. We found that approximately $70 \%$ of the MUC5AC-associated genes identified in airway epithelium [16] are also expressed in the lung fibroblasts [18]. Finally, the MUC5AC-associated genes may be secondary targets of miR-134-5p. Our recent studies have shown that CXCL8 produced by lung fibroblasts promotes epithelial mucus production [8] and that CXCL8 release is increased upon co-culture of fibroblasts and epithelial cells [20]. In line with that report, we observed in this study that CXCL8 expression is negatively correlated with miR-134-5p (supplementary table S6).

Another key miRNA identified in our miRNA-mRNA networks was miR-146a-5p, which was lower expressed with CMH. Notably, miR-146a-5p was previously reported to be involved in airway inflammation associated with smoking and COPD [10] and to inhibit MUC5AC production in vitro [21]. We recently found miR-146a-5p to be higher expressed in non-COPD lung fibroblasts upon co-culture with bronchial epithelial cells, while there was significantly less increase in COPD fibroblasts, suggesting a role for miR-146a-5p in disturbed epithelial-fibroblast crosstalk in COPD [22]. Our present findings suggest that miR-146a-5p may also be involved in mucus hypersecretion via epithelial-fibroblast crosstalk; however, it is not yet clear via which target gene nor in which cell type it has its main effect on CMH. Further mechanistic studies using co-culture or lung organoid models could help in elucidating the $\mathrm{CMH}$-related function of miR-146-5p.

The last key miRNA-mRNA cluster identified was the let-7 family, which was higher expressed in association with $\mathrm{CMH}$. The members of the let-7 family shared several potential targets associated with $\mathrm{CMH}$, of which EDN1, NKD1, PDGFB, COL4A1 and COL4A2 are of particular interest. EDN1 is localised in submucosal glands and stimulates mucus secretion from serous and mucus cells in mucosal explant culture [23]. NKD1 is an antagonist of the WNT/B-catenin signalling pathway [24], which plays an important role in respiratory epithelial differentiation [25] and, of which, specific components (e.g. LEF1) have been reported to regulate MUC5AC production $[8,26]$. PDGFB is involved in airway remodelling [27] and epithelial-fibroblast crosstalk [28]. In addition, COL4A1 and COL4A2 were also identified in a larger cluster of collagen genes in the $\mathrm{CMH}$ interaction networks and are essential components of basement membranes [29]. Basement membrane thickness is associated with an increase of submucosal glands and central airway remodelling in asthma [30], but its role in linking airway remodelling with $\mathrm{CMH}$ in COPD remains to be investigated. Of note, various $\mathrm{CMH}$-associated targets of let-7a-5p/ let-7d-5p/let-7f-5p (EDA, LIX1L, MAPK11 and NME4) have been validated with next-generation sequencing [31], supporting our findings.

Furthermore, the $\mathrm{CMH}$-associated genes we identified may be involved in several other biological processes, including cilium development and function, neurohormonal activities, cyclic nucleotide metabolism and signalling, and ion transport. Cilia function and movement is important for mucus clearance [32] and impaired cilia function has been observed in COPD airways [33, 34]. The movement of cilia is dependent, at least partially, on cyclic nucleotides and in particular on cAMPs [35]. In addition, active ion transport, such as that of $\mathrm{Ca}^{2+}, \mathrm{Na}^{+}$and $\mathrm{Cl}^{-}$, plays an important role in regulating mucus viscosity and mucociliary clearance $[36,37]$, and both $\mathrm{Ca}^{2+}$ and $\mathrm{Na}^{+}$can be transported through cyclic nucleotide-gated channels [38]. Notably, previous studies have suggested that neurohormonal signalling regulates cyclic guanosine monophosphate (cGMP) induced mucin secretion [39] and the expression of GDNF, the gene encoding for a neurotrophic factor involved in lung development, is associated with $\mathrm{CMH}$ in COPD [40]. We also generated gene-interaction networks for $\mathrm{CMH}$ and identified KRAS, EDN1, PRKAR2A, GSK3B and POLR2H as hub genes and potential regulators of CMH. Next to KRAS and EDN1, $G S K 3 B$ is also of interest as it is a pleiotropic signalling molecule that regulates WNT/ $\beta$-catenin signalling (the pathway that has been shown to induce mucus cell metaplasia in a mouse model) [25].

One of the limitations of this study is the relatively small dataset that we used to explore the miRNA and mRNA changes associated with $\mathrm{CMH}$ and the consequent use of a lenient FDR cut-off. To support our initial findings, several approaches were used. First, we used GSEA to demonstrate the enrichment of MUC5AC-associated genes [16] among the genes higher expressed with CMH. This confirmed the relationship between our $\mathrm{CMH}$ definitions and MUC5AC expression. In addition, we demonstrated 
significant enrichment of the $\mathrm{CMH}$-associated genes identified in the microarray dataset among the $\mathrm{CMH}$-associated genes identified in the RNA-sequencing dataset. The replication of our findings in a different patient cohort would be informative; however, to the best of our knowledge, there are no other datasets in which both miRNA/mRNA profiles and $\mathrm{CMH}$ definitions are available, reflecting an urgent need for more studies on this topic.

For the in vitro studies, we decided to determine the expression of our candidate miRNAs in PAECs and PAFs, as they are present in airway wall biopsies and important cells in the pathogenesis of COPD. As our findings were derived from biopsies, miRNA changes in other cell types (e.g. inflammatory cells or smooth muscle cells) may have contributed to our findings. In fact, this could explain the lack of expression of miR-500a-3p and miR-1207-5p in PAECs and PAFs, and validation in other cell types and co-culture studies is warranted in future work. Furthermore, it will be of interest for future investigations to compare PAECs from patients with $\mathrm{CMH}$ and those without $\mathrm{CMH}$, which was not possible in the current study. In addition, we focused on the 10 miRNAs that were significantly correlated with mRNA expression in the same subjects in this study. This does not imply that the other 10 miRNAs were not relevant to $\mathrm{CMH}$, since miRNAs not only regulate gene translation by degradation but also by inhibiting translation [9].

Collectively, we identified three key miRNA-mRNA clusters for CMH: miR-134-5p, miR-146a-5p and the let-7 family, as well as their associated potential target genes. The let-7 and miR-134-5p clusters are connected to the $\mathrm{CMH}$ gene expression networks via the potential key regulatory genes KRAS and EDN1. Furthermore, we identified pathways and biological processes, including MUC5AC-associated genes, in which these key miRNA-mRNA clusters are likely to be involved. Future studies involving co-cultures of epithelial cells with fibroblast and other cell types are required to further unravel the functional role of these key genes and miRNAs, and to establish whether they represent potential therapeutic targets for CMH.

Acknowledgements: The authors would like to thank Anita Spanjer (University of Groningen, Groningen, The Netherlands) for providing RNA samples from human primary airway fibroblasts, Jeunard Glenn Boekhoudt (University of Groningen, Groningen, The Netherlands) for the analyses of the RNA sequencing data and Margaret Neighbors (Genentech, San Francisco, CA, USA) for securing funding from Genentech for the RNA sequencing analyses.

Author contributions: H. Tasena, A. Faiz, W. Timens, M.N. Hylkema, R. Gosens, D.S. Postma, M. van den Berge, I.H. Heijink and C-A. Brandsma contributed to the study concept and design. P.S. Hiemstra, M. van den Berge and W. Timens coordinated patient inclusion and data collection for GLUCOLD. M. van den Berge and A. Spira organised and performed the microarrays. G.W. Tew and M.A. Grimbaldeston organised and performed the RNA sequencing. H. Tasena and J. Noordhoek conducted in vitro laboratory work under the supervision of I.H. Heijink and C-A. Brandsma. H. Tasema and A. Faiz performed the microarray data analysis under the supervision of M. van den Berge and C-A. Brandsma. H. Tasena, A. Faiz, M. van den Berge, I.H. Heijink and C-A. Brandsma analysed and interpreted data, and drafted the manuscript. H. Tasena, A. Faiz, W. Timens, J. Noordhoek, M.N. Hylkema, R. Gosens, P.S. Hiemstra, A. Spira, D.S. Postma, G.W. Tew, M.A. Grimbaldeston, M. van den Berge, I.H. Heijink and C-A. Brandsma critically read and revised the manuscript. All authors read, reviewed and approved the final manuscript.

Conflict of interest: W. Timens reports receiving an unrestricted grant from Merck to his institution during the conduct of the study; a consultancy fee to his institution from Pfizer, lecture fees to his institution from GSK, Chiesi and Boehringer Ingelheim, lecture and consultancy fees and travel costs to his institution from Roche Diagnostics/Ventana, a grant from the Dutch Asthma Fund, travel costs to his institution from Biotest, consultancy and course fees to his institution from Merck Sharp Dohme, lecture/course and consultancy fees to his institution from Novartis, personal fees from Lilly Oncology, outside the submitted work. R. Gosens reports receiving grants from Boehringer Ingelheim, Chiesi Farmaceutici and Aquilo, is a cofounder of Aquilo, and reports receiving grants from and has served on an advisory board for Holaira, outside the submitted work. P. Hiemstra reports receiving grants from Galapagos N.V. and Boehringer Ingelheim, outside the submitted work. A. Spira has acted as a consultant for and holds stock options in Veracyte Inc, and has acted as a consultant for Janssen Pharma, outside the submitted work. D.S. Postma reports that the University of Groningen has received money regarding a grant for research from AstraZeneca, Chiesi, Genentech, GSK and Roche. Fees for consultancies were given to the University of Groningen by AstraZeneca, Boehringer Ingelheim, Chiesi, GSK, Takeda and TEVA. G.W. Tew is an employee of Genentech Inc. M.A. Grimbaldeston is an employee of Genentech Inc. M. van den Berge reports grants paid to the University of Groningen from AstraZeneca, TEVA, GSK and Chiesi, outside the submitted work.

Support statement: This study was funded by a non-restricted grant from Stichting Astma Bestrijding, the Netherlands Asthma Foundation. Funding information for this article has been deposited with the Crossref Funder Registry.

\section{References}

1 Corhay JL, Vincken W, Schlesser M, et al. Chronic bronchitis in COPD patients is associated with increased risk of exacerbations: a cross-sectional multicentre study. Int J Clin Pract 2013; 67: 1294-1301.

2 Burgel P, Nesme-Meyer P, Chanez P, et al. Cough and sputum production are associated with frequent exacerbations and hospitalizations in COPD subjects. Chest 2009; 135: 975-982.

Ramos FL, Krahnke JS, Kim V. Clinical issues of mucus accumulation in COPD. Int J COPD 2014; 9: 139-150. Burgel PR. Chronic cough and sputum production: a clinical COPD phenotype? Eur Respir J 2012; 40: 4-6. Kirkham S, Sheehan JK, Knight D, et al. Heterogeneity of airways mucus. Biochem J 2002; 546: 537-546. 
6 Caramori G, Di Gregorio C, Carlstedt I, et al. Mucin expression in peripheral airways of patients with chronic obstructive pulmonary disease. Histopathology 2004; 45: 477-484.

7 Albers S, Thiebes AL, Gessenich KL, et al. Differentiation of respiratory epithelium in a 3-dimensional co-culture with fibroblasts embedded in fibrin gel. Multidiscip Respir Med 2015; 11: 6.

8 Spanjer AIR, Menzen MH, Dijkstra AE, et al. A pro-inflammatory role for the Frizzled-8 receptor in chronic bronchitis. Thorax 2016; 71: 312-322.

9 Engels BM, Hutvagner G. Principles and effects of microRNA-mediated post-transcriptional gene regulation. Oncogene 2006; 25: 6163-6169.

10 Osei ET, Florez-Sampedro L, Timens W, et al. Unravelling the complexity of COPD by microRNAs: it's a small world after all. Eur Respir J 2015; 46: 807-818.

11 Schembri F, Sridhar S, Perdomo C, et al. MicroRNAs as modulators of smoking-induced gene expression changes in human airway epithelium. Proc Natl Acad Sci U S A 2009; 106: 2319-2324.

12 Lapperre TS, Sont JK, van Schadewijk A, et al. Smoking cessation and bronchial epithelial remodelling in COPD: a cross-sectional study. Respir Res 2007; 8: 85.

13 Lapperre TS, Postma DS, Gosman MME, et al. Relation between duration of smoking cessation and bronchial inflammation in COPD. Thorax 2006; 61: 115-121.

14 Heijink IH, De Bruin HG, Dennebos R, et al. Cigarette smoke-induced epithelial expression of WNT-5B: implications for COPD. Eur Respir J 2016; 48: 504-515.

15 Brandsma C-A, Timens W, Jonker MR, et al. Differential effects of fluticasone on extracellular matrix production by airway and parenchymal fibroblasts in severe COPD. Am J Physiol Lung Cell Mol Physiol 2013; 305: L582-L589.

16 Wang G, Xu Z, Wang R, et al. Genes associated with MUC5AC expression in small airway epithelium of human smokers and non-smokers. BMC Med Genomics 2012; 5: 21.

17 Ross AJ, Dailey LA, Brighton LE, et al. Transcriptional profiling of mucociliary differentiation in human airway epithelial cells. Am J Respir Cell Mol Biol 2007; 37: 169-185.

18 Ong J, Timens W, Rajendran V, et al. Identification of transforming growth factor-beta-regulated microRNAs and the microRNA-targetomes in primary lung fibroblasts. PLoS One 2017; 12: e0183815.

19 Zhang J, Li S, Mi S, et al. Exosome and exosomal microRNA: trafficking, sorting, and function. Genomics Proteomics Bioinformatics 2015; 13: 17-24.

20 Osei ET, Noordhoek JA, Hackett TL, et al. Interleukin-1 $\alpha$ drives the dysfunctional cross-talk of the airway epithelium and lung fibroblasts in COPD. Eur Respir J 2016; 48: 359-369.

21 Zhong T, Perelman JM, Kolosov VP, et al. MiR-146a negatively regulates neutrophil elastase-induced MUC5AC secretion from 16HBE human bronchial epithelial cells. Mol Cell Biochem 2011; 358: 249-255.

22 Osei ET, Florez-Sampedro L, Tasena H, et al. MiR-146a-5p plays an essential role in the aberrant epithelial-fibroblast crosstalk in COPD. Eur Respir J 2017; 49: 1602538.

23 Mullol J, Chowdhury BA, White MV, et al. Endothelin in human nasal mucosa. Am J Respir Cell Mol BioI 1993; 8: 393-402.

24 Van Raay TJ, Coffey RJ, Solnica-Krezel L. Zebrafish Naked1 and Naked2 antagonize both canonical and non-canonical Wnt signaling. Dev Biol 2007; 309: 151-168.

25 Mucenski ML, Nation JM, Thitoff AR, et al. $\beta$-Catenin regulates differentiation of respiratory epithelial cells in vivo. Am J Physiol Lung Cell Mol Physiol 2005; 289: L971-L979.

26 Young HWJ, Williams OW, Chandra D, et al. Central role of Muc5ac expression in mucous metaplasia and its regulation by conserved 5' elements. Am J Respir Cell Mol Biol 2007; 37: 273-290.

27 Yamashita N, Sekine K, Miyasaka T, et al. Platelet-derived growth factor is involved in the augmentation of airway responsiveness through remodeling of airways in diesel exhaust particulate-treated mice. J Allergy Clin Immunol 2001; 107: 135-142.

28 Li DQ, Tseng SC. Three patterns of cytokine expression potentially involved in epithelial-fibroblast interactions of human ocular surface. J Cell Physiol 1995; 163: 61-79.

29 Pöschl E, Schlötzer-Schrehardt U, Brachvogel B, et al. Collagen IV is essential for basement membrane stability but dispensable for initiation of its assembly during early development. Development 2004; 131: 1619-1628.

30 James AL, Maxwell PS, Pearce-Pinto G, et al. The relationship of reticular basement membrane thickness to airway wall remodeling in asthma. Am J Respir Crit Care Med 2002; 166: 1590-1595.

31 Helwak A, Kudla G, Dudnakova T, et al. Mapping the human miRNA interactome by CLASH reveals frequent noncanonical binding. Cell 2013; 153: 654-665.

32 Knowles MR, Boucher RC. Mucus clearance as a primary innate defense mechanism for mammalian airways. J Clin Invest 2002; 109: 571-577.

33 Rogers DF. Mucociliary dysfunction in COPD: effect of current pharmacotherapeutic options. Pulm Pharmacol Ther 2005; 18: 1-8.

34 Smaldone GC, Foster MW, O'Riordan TG, et al. Regional impairment of mucociliary clearance in chronic obstructive pulmonary disease. Chest 1993; 103: 1390-1396.

35 Wyatt TA, Forge MA, Sisson JH. Ethanol stimulates ciliary beating by dual cyclic nucleotide kinase activation in bovine bronchial epithelial cells. Am J Pathol 2003; 163: 1157-1166.

36 González C, Droguett $\mathrm{K}$, Rios $\mathrm{M}$, et al. TNF $\alpha$ affects ciliary beat response to increased viscosity in human pediatric airway epithelium. Biomed Res Int 2016; 2016: 3628501.

37 Tarran R, Grubb BR, Gatzy JT, et al. The relative roles of passive surface forces and active ion transport in the modulation of airway surface liquid volume and composition. J Gen Physiol 2001; 118: 223-236.

38 Kaupp UB, Seifert R. Cyclic nucleotide-gated ion channels. Physiol Rev 2002; 82: 769-824.

39 Shelhamer JH, Marom ZVI, Kaliner M, et al. Immunologic and neuropharmacologic stimulation of mucous glycoprotein release from human airways in vitro. J Clin Invest 1980; 66: 1400-1408.

40 Dijkstra AE, Boezen HM, Van Den Berge M, et al. Dissecting the genetics of chronic mucus hypersecretion in smokers with and without COPD. Eur Respir J 2015; 45: 60-75. 